\title{
Development of Blast Resistant Dubraj by Introgression of Resistant Genes through Marker Assisted Selection
}

\author{
Shinde Umesh Dnyaneshwar ${ }^{1 *}$, Toshy Agrawal ${ }^{1}$, Ashish Pradhan ${ }^{2}$, Tanvi Kadu ${ }^{1}$, \\ Anil S. Kotasthane ${ }^{2}$ and Satish B. Verulkar ${ }^{1}$ \\ ${ }^{I}$ Department of Plant Molecular Biology and Biotechnology, COA, IGKV, \\ Raipur (C.G.), India \\ ${ }^{2}$ Department of Plant Pathology, COA, IGKV, Raipur (C.G.), India \\ *Corresponding author
}

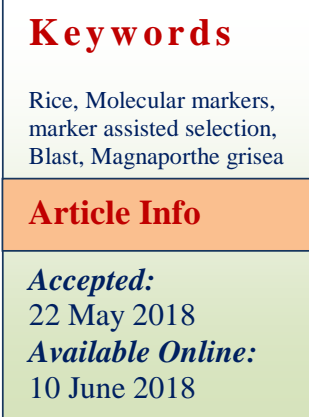

\section{Introduction}

Rice is major crop in Indian agricultural system not only due to economical aspects but also as staple food. It serve as major dietary component in Indian meal. Increase in the

\section{A B S T R A C T}

Despite the fact that rising global population requires increased crop production, the rate of increase in crop yields is currently declining. The traits related to yield, stability and sustainability should be a major focus of plant breeding efforts. These traits include durable disease resistance, abiotic stress tolerance along with nutrient and water use efficiency. The use of DNA markers in plant breeding is called marker assisted selection (MAS). So far, about 101 major blast genes have been identified, many of them have been mapped cloned on different rice chromosomes, and tightly linked DNA markers have been developed. So, blast resistance genes can be combined using MAS in a single genetic background to develop rice cultivars with broad-spectrum durable resistance to blast. In this research work, we have introgressed two genes Pi1 and Pi2 in local variety of Chhattisgarh dubraj. Dubraj is extremely popular aromatic short medium grain variety in Chhattisgarh and Madhya Pradesh region. PR-122 is used as donor for resistant gene to introgress blast genes $\mathrm{Pi}-1$ \& Pi-2. The cross was attempted and progenies were obtained in Kharif 2016 season. Out of 29 plants 9 plants were selected for further screening. Progenies were screened phenotypically as well as genotypically for blast resistance for three consecutive seasons (Kharif 2016, Summer 2017 \& Kharif 2017). During kharif 2017, 9 plants showing blast resistance both phenotypically as well as genotypically were sown in summer 2018. Forty seven plants with positive correlation between phenotype and genotype for blast resistance as observed in Summer 2017, were taken up for further generation advancement and screening during Kharif- 2018. Combination of conventional and marker assisted selection approach will provide opportunities for breeders to develop high yielding, stress tolerant and better quality rice variety.

yield of crops have to be kept in pace with the increasing population, for which cultivation of high yielding varieties is required, give high inputs like increased fertilizers doses and insecticides have altered the dynamics of pest and diseases and significantly increases their 
incidence in recent years. Several diseases are reported in rice cropping system among which blast is an important disease accounting for major yield loss (Zhang et al., 2015) and it is endemic to several rice growing states (Production Oriented Survey. 2008). To minimize the problem, durable and resistance varieties need to be introduced into disease prone areas (Sundaram et al., 2014). Till date 101 blast resistant genes (Rajashekara et al., 2014) and 350 Quantitative Trait Loci (QTLs) have been identified (Sharma et al., 2012).

In Chhattisgarh and Madhya Pradesh region, Dubraj is an extremely popular aromatic, short medium grain variety with intermediate amylose content. However it is highly susceptible to blast disease which restricts its cultivation in disease prone areas. Several chemical fungicides like Tricyclazole (22\%), hexaconazole $(3 \%)$ are used to repress the blast infestation, which is expensive \& responsible for environmental hazards. Most serious problem of use of chemicals is the reappearance of resistant races of pathogen. Due to availability of several resistance genes for blast, pyramiding of such genes into dubraj is considered as an excellent strategy to develop resistant variety. Breeding for hostplant resistance is considered as most acceptable strategy for disease and pest management. Through conventional breeding method breeders have developed many blast resistance varieties (Miah et al., 2013).

In conventional breeding programme different methods (pedigree method, backcrossing method, recurrent selection) have been frequently used. However, gene pyramiding through conventional screening is difficult and likely to be impossible because of the dominance and epistasis effect of resistance genes and lack of high precision phenotyping approaches. The abundance of closely linked or within gene molecular marker enables gene pyramiding easier (Zhao et al., 2014).
In the present study we targeted to pyramid two major blast genes Pi1 and Pi2 into dubraj variety from PR-122 donor through Marker Assisted Selection approach.

\section{Materials and Methods}

PR-122 is medium maturity, high yielding non-basmati variety released by the State Variety Approval Committee for general cultivation in the Punjab during 2013. It has been developed from the cross PR-108/IRRI76//PR-106-P1 through pedigree method of selection and used as donor parent for resistant genes. While dubraj is an aromatic short to medium grain rice which is a traditional Indian variety famous in Madhya Pradesh and Chhattisgarh was used as recurrent parent for introgression of blast gene. Populations derived from cross between PR-122 and Dubraj were selected through targeted genotyping and phenotyping analysis.

\section{Method}

The recurrent parent Dubraj was crossed with donor PR-122 in kharif 2014. Resulting progenies were phenotypically and genotypically screened for resistance towards Blast. Selected plants were subsequently used for advancement of generation during next cropping season(s) kharif 2016, summer 2017 and kharif 2017. The progenies which gave positive results in both genotype and phenotype were selected for further research.

\section{Targeted genotyping}

For targeted introgression of Pi1 and Pi2 resistant genes into dubraj a stepwise marker assisted selection strategy was adopted (Fig.1). Recurrent parent dubraj was crossed with donor PR-122 to obtain progenies. These progenies were confirmed for their hybridity or presence of gene of interest with a set of SSR markers RM224 and RM 7311 for Pi1 
and $\mathrm{Pi} 2$ respectively. These markers are firstly subjected for parental polymorphism which shows clear polymorphism between parents and further used on progeny selection. DNA from selected lines were extracted using modified CTAB method and used for PCR amplification at initial denaturation of $95^{\circ} \mathrm{C}$ for 5 minutes with denaturation of 30 seconds, annealing at $55^{\circ} \mathrm{C}$ for $30 \mathrm{~s}$ and extension at $72^{\circ} \mathrm{C}$ for $1 \mathrm{~min}$ with 30 cycles anf final extension at $72^{\circ} \mathrm{C}$ for 7 minutes. Amplified products will be resolved on 5\% PAGE.

\section{Screening of Disease reaction}

The phenotypic screening of progenies along with parents was done at Ambikapur district of Chhattisgarh. Which is the hotspot for blast disease. The plants were evaluated and scored as per IRRI-SES scale.

\section{Results and Discussion}

\section{Confirmation of polymorphism of gene specific marker}

The DNA from recurrent parent dubraj and from donor parent PR-122 were isolated to determine the polymorphism of markers. The primer pair for Pi1 (RM 224) amplified fragments of 150bp in resistant parent PR-122 while in susceptible parent dubraj it amplified 140bp fragment. With respect to the primer pair for Pi2 (RM7311) amplified fragment of 150bp in resistant parent PR-122 and 140bp fragment in susceptible parent dubraj.

Marker assisted introgression of Blast resistance

It was observed that in Dubraj cross [(DxDPB)x(DxPR122)] out of 8 lines consisting of 29 plants, only 2 lines with 9 plants; two of them shows presence of both resistance gene while 2 and 3 plant shows heterozygous condition for $\mathrm{Pi} 1$ and $\mathrm{Pi} 2$ resistant genes respectively. Among these 9 plants these markers are linked to resistance genes and scoring was done based on the banding pattern as per their respective parents. Those bands likely to be that of resistant and susceptible parents were score as $\mathrm{R}$ and $\mathrm{S}$ respectively. While plants showing bands from both the parents were scored as Heterozygous $(\mathrm{H})$. Seeds of selected 9 plants of [(DxDPB)x(DxPR122)] cross from Kharif 2016 were then further sown as 9 lines (with 78 plants).

Out of 78 plants, 13 plants possesses both resistant gene, while 18 and 25 plants shows heterozygous condition for Pi1 and $\mathrm{Pi} 2$ gene respectively. 43 and 39 plants out of 78 shows absence of Pi1 and Pi2 respectively. Total 47 plants were selected in summer 2017 through genotypic scoring of banding pattern with linked marker as well as with phenotyping. These selected 47 plants were further sown for generation advancement in Kharif 2017. In kharif 2017out of 108 progenies 10 progenies shows presence of both resistant genes while 4 progenies shows Pi1 gene. 36 and 39 progenies shows heterozygous condition for Pi1 and Pi2 gene respectively. While 58 and 59 progenies were lack of any of the resistant gene. (Table 2, Fig. 2). Though the donor plant PR-122 contains two resistance genes (Pi1 and Pi2). Two PCR based markers RM224 and RM7311 were able to identify double positive plants precisely without any false positive result the same result were observed in Arunakumari et al., (2016).

In this study no negative interaction was observed between genes conferring resistance against blast. Similar results were observed by Sundaram et al., (2008) and Hari et al., (2013). Further no negative effects were found due to presence of resistance gene in any pyramided lines, since donor parents PR-122 was improved variety with desirable agro morphological and other quality character. 
Fig.1 Steps involved for developing resistance against blast in Dubraj

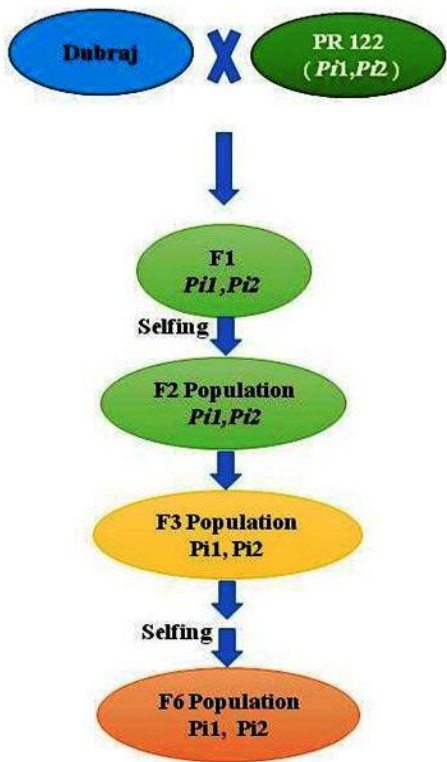

Fig.2 PCR amplification of population derived from Dubraj x PR-122 cross using linked markers for Pi1 and Pi2

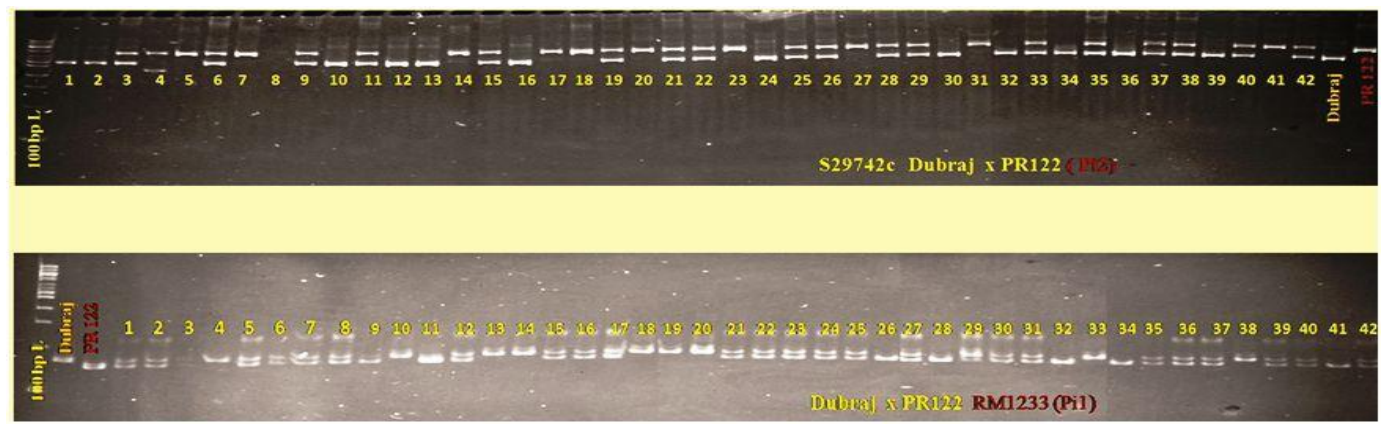

Fig.3 Disease infestation on parents

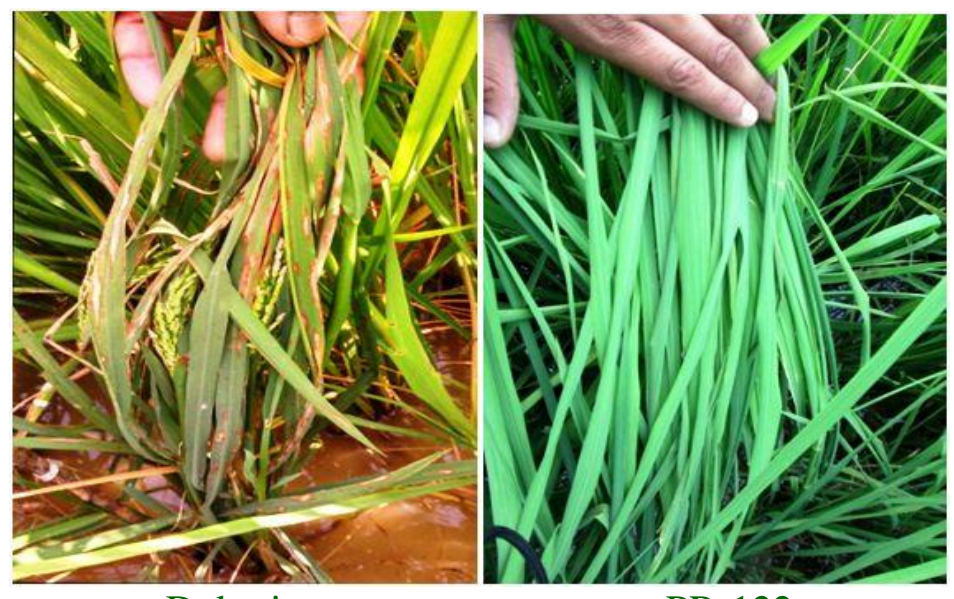

Dubraj

PR-122 
Table.1 Markers used for foreground blast resistance genes in marker-assisted selection

\begin{tabular}{|c|c|c|c|c|}
\hline S. No. & \multicolumn{2}{|l|}{ Marker } & Sequence & Resistance gene \\
\hline \multirow[t]{2}{*}{1.} & \multirow[t]{2}{*}{ RM224 } & Forward & ATCGATCGATCTTCACGAGG & \multirow[t]{2}{*}{ Pi 1} \\
\hline & & Reverse & TGCTATAAAAGGCATTCGGG & \\
\hline \multirow[t]{2}{*}{2.} & \multirow[t]{2}{*}{ RM 7311} & Forward & AGTGGTCGTTGAACTCGGAG & \multirow[t]{2}{*}{ Pi 2} \\
\hline & & Reverse & TCGTGGCGCCTTTAATCTC & \\
\hline
\end{tabular}

Table.2 Result of Genotyping and Phenotyping

\section{D x RPB X D x PR-122}

\begin{tabular}{|l|l|l|l|l|l|l|l|l|l|l|l|} 
& Pi 1 & \multicolumn{1}{c|}{ Pi 2} & \multicolumn{3}{|c|}{ Phenotype } & \\
& R & H & S & AB & R & H & S & AB & R & S & TOTAL \\
\hline KH 16 & 2 & 2 & 24 & 1 & 2 & 3 & 24 & 0 & 9 & 20 & 29 \\
\hline S 17 & 17 & 18 & 43 & 0 & 13 & 25 & 39 & 1 & 60 & 18 & 78 \\
\hline KH 17 & 14 & 36 & 58 & 0 & 10 & 39 & 59 & 0 & 91 & 17 & 108 \\
\hline
\end{tabular}

Note:- $\mathrm{R}$ represents Resistant progeny, S- susceptible, H- Hybrid, AB- Absent, KH 16- Kharif 2016, KH 17Kharif 2017, S17- Summer 2017, D- Dubraj, RPB- RP Bio, S17- Safri 17

The similar outcome were observed by Arunakumari et al., (2016) Through deployment of Marker assisted selection with precise phenotype based selection we are able to recover desirable grain and plant type as like to the recurrent parent dubraj.

\section{Screening of Blast reaction}

The phenotypic screening of progenies along with parents was done at Ambikapur. The plants were evaluated and scored as per IRRISES scale. In kharif 2016 season total 29 progenies were screened for blast reaction at ambikapur out of it 9 plants were shows resistant phenotype with mean score of 0 to 3 . While 20 plants shows susceptible phenotype. In summer 2017 season total 78 plants were screened out of it 60 plants shows resistant phenotype with mean score of 0 to 3 while 18 plants shows susceptible phenotype. Out of 108 plants 91 shows resistant and 17 shows susceptible phenotype in kharif 2017 season. All the lines selected through selected genotyping with PCR based markers shows high level of resistance in field condition.
This result were states that no any crossing over event were happened between marker and the resistant gene. This result were also observed by Arunakumari et al., (2016). We were successful in identifying superior recombination's for two blast resistant genes (Pi1 and Pi2) in homozygous as well as in heterozygous conditions. The pyramided genotypes can be further be used for multilocation testing to be released as variety in the country or be used as potential blast resistance donors. These blast pyramided lines are expected to have a high impact on the yield stability and sustainability of rice production.

\section{Acknowledgement}

The financial assistance from Department of Plant Molecular Biology and Biotechnology Indira Gandhi Krishi Vishwavidyalaya, Raipur is gratefully acknowledged.

\section{References}

Arunakumari, K. et al., (2016) 'MarkerAssisted Pyramiding of Genes 
Conferring Resistance Against Bacterial Blight and Blast Diseases into Indian Rice Variety MTU1010', Rice Science, 23(6), pp. 306-316. doi: 10.1016/j.rsci.2016.04.005.

Hari, Y. et al., (2013) 'Marker- assisted introgression of bacterial blight and blast resistance into IR 58025B, an elite maintainer line of rice', Plant Breeding. Wiley Online Library, 132(6), pp. 586594.

Miah, G. et al., (2013) 'Blast resistance in rice: a review of conventional breeding to molecular approaches', Molecular biology reports. Springer, 40(3), pp. 2369-2388.

Rajashekara, H. et al., (2014) 'Inheritance of blast resistance and its allelic relationship with five major $\mathrm{R}$ genes in a rice landrace "Vanasurya.", Indian Phytopathol, 67, pp. 365-369.

Sharma, T. R. et al., (2012) 'Rice blast management through host-plant resistance: retrospect and prospects', Agricultural Research. Springer, 1(1), pp. 37-52.

Sundaram, R. M. et al., (2008) 'Marker assisted introgression of bacterial blight resistance in Samba Mahsuri, an elite indica rice variety', Euphytica. Springer, 160(3), pp. 411-422.

Sundaram, R. M. et al., (2014) 'Update on bacterial blight of rice: fourth international conference on bacterial blight'. Springer.

Zhang, S. Y. et al., (2015) 'Investigation of rice virusdiseases and analysis of the molecular variation of RSV isolatesin the main rice-growing areas of Yunnan Province from 2013 to2014', Chin J Rice Sci, 29(5), pp. 535-545.

Zhao, G. Z. et al., (2014) 'Identification and analysis on the rice blast resistance genePi-ta in wild rice from Yunnan, China', Chin J Rice Sci, 28(6), pp. 675680 .

\section{How to cite this article:}

Shinde Umesh Dnyaneshwar, Toshy Agrawal, Ashish Pradhan, Tanvi Kadu, Anil S. Kotasthane and Satish B. Verulkar. 2018. Development of Blast Resistant Dubraj by Introgression of Resistant Genes through Marker Assisted Selection. Int.J.Curr.Microbiol.App.Sci. 7(06): 3113-3118. doi: https://doi.org/10.20546/ijcmas.2018.706.365 